\title{
Turovsky 0. ESTIMATION OF THE POSSIBILITIES OF THE COMBINED SYNCHRONIZATION SYSTEM WITH OPEN-LINK TO MINIMIZE THE DISPERSION OF THE PHASE ERROR WHEN TRACKING THE CARRIER FREQUENCY UNDER THE CONDITIONS OF THE INFLUENCE OF ADDITIVE NOISE
}

The object of the article is the process of evaluating the possibilities of a combined synchronization system with open (compensating) connection to minimize the phase error variance under the influence of additive Gaussian noise.

The issue of improving the quality of the phase synchronization system is a constant topical scientific task and in a number of studies is solved by including the method of developing mathematical models and creating on their basis the appropriate optimal schemes for building these systems. The final stage of development and implementation of such mathematical models in technical solutions and synchronization schemes based on them is the assessment of the limit possibilities of these schemes to minimize the variance of the phase error in different types of noise.

The paper evaluates the possibilities of a combined synchronization system with open (compensating) connection to minimize the phase error dispersion under the influence of additive Gaussian noise by selecting the parameters of the components of the circuit of this connection.

In order to evaluate the work, mathematical dependencies have been developed and refined, which allow to determine the variance of the phase error under the influence of additive Gaussian noise and the corresponding algorithm for its determination is presented.

The evaluation results presented in the work showed that the value of the error variance for the combined synchronization system is much smaller than for the closed synchronization system with any choice of the parameters of the latter. The effect of reducing the phase error variance by introducing a broken link at a given level of influence depends on the noise signal level and the phase modulation index of the input signal. When using as a simple broken link a frequency discriminator included in the circuit of the combined synchronization system in parallel, the minimum dispersion of the phase error when the noise level rises to a certain critical limit is limited and this broken link loses its effectiveness.

Keywords: carrier frequency synchronization, combined synchronization system, phase error variance, additive Gaussian noise.

Received date: 10.04.2020

Accepted date: 22.05.2020

Published date: 31.08 .2020
Copyright (c) 2020, Turovsky O. This is an open access article under the CC BY license (http://creativecommons.org/licenses/by/4.0)

\section{Introduction}

The quality of modern communication systems depends on various factors and conditions, including the influence of a number of external disturbances and internal noise. For example, in space communication lines the main external perturbations are additive Gaussian noise and Doppler frequency shifts [1,2]. The task of improving the quality of communication systems is to ensure the necessary, as a rule, high accuracy of their operation in constant and transient modes.

In turn, the successful solution of the problem of improving the efficiency of communication systems largely depends on the quality of operation under the influence of these perturbations and noise of individual systems and devices that are part of them. As one of the main systems which is a part of various radio engineering devices of equipment of communication, radar and management and also in the device of exact magnetic record the system of phase synchronization is widely implemented. For example, in phase-coherent telecommunications and control systems, such systems are used to restore the carrier and clock frequencies and for coherent demodulation of analog and digital signals with angular modulation [3].

The solution of the scientific problem of improving the efficiency of the synchronization system can be achieved by various methods. Including conducting relevant research on the creation of combined synchronization systems, which include an open link.

In turn, evaluation of the possibilities of such systems to minimize the variance of the phase error is a separate scientific problem, the solution of which determines the relevance of the research conducted in this work. 


\section{The object of research and its technological audit}

The object of research is the process of evaluating the possibilities of a combined synchronization system with an open (compensating) connection to minimize the phase error variance under the influence of additive Gaussian noise.

The operation of synchronization systems is characterized directly by the influence of additive fluctuation noise, perturbation of useful angular modulation (in the case of carrier frequency filtering), phase and frequency jumps and others [1, 3]. In some cases, it is necessary to ensure high accuracy of the specified system in constant and transient modes under the influence of these noises. And synchronization systems operating in such conditions should be characterized by low phase error variance and high speed.

The issue of improving the quality of the phase synchronization system is a constant topical scientific task and in a number of studies is solved including the method of creating appropriate optimal schemes for its construction in the direction of minimizing the phase error variance and at the same time ensuring high speed.

It is obvious that these schemes solve the problem of minimizing the phase error through the development of scientifically sound optimal construction schemes that operate on the basis of developed mathematical models. These mathematical models, in turn, must take into account the parameters of all functional components and elements of the specified scheme of the synchronization system. The final stage of development and implementation of such mathematical models in technical solutions and synchronization schemes based on them is the evaluation of the limit possibilities of these schemes to minimize the phase error variance by selecting the appropriate parameters of components and functional components of synchronization systems.

The final stage of development and implementation of such mathematical models in technical solutions and synchronization schemes built on their basis is the solution of a number of problems, namely:

- assessment of the marginal possibilities of these schemes

to minimize the variance of the phase error;

- selection and substantiation of parameters of constituent elements and functional links of the scheme of construction of synchronization systems in the direction of minimization of dispersion of a phase error in the course of monitoring of the carrier frequency.

\section{The aim and objectives of research}

The aim of research is to evaluate the possibilities of a combined synchronization system with open (compensating) connection in relation to the influence of the parameters of the components of the scheme of such a connection to minimize the phase error variance of the synchronization system under the influence of additive Gaussian noise.

To achieve this aim it is necessary:

1. To develop appropriate mathematical dependencies that allow to determine the variance of the phase error of the combined synchronization system under the influence of additive Gaussian noise.

2. To use mathematical modeling methods to evaluate the influence of the parameters of the open link on minimizing the variance of the phase error of the combined synchronization system from the signal level of the additive Gaussian noise.

\section{Research of existing solutions to the problem}

A number of works are devoted to the construction of synchronization circuits and evaluation of their possibilities to minimize the phase error variance.

The paper [1] presents the results of research on the analysis and formalization of the description of synchronization devices such as solution-controlled and non-solutioncontrolled. The dependencies for the variance of the maximum plausible estimate of the synchronization device on the signal-to-noise ratio, which are designed for a system without feedback on the solution, are given. The variances of the variance of the maximum plausible estimation of the synchronization device from the signal-to-noise ratio and the dependencies of the deviation of the synchronization error from the signal-to-noise ratio at the output of the phase detector presented in this work are designed for the circuit without feedback. Such a scheme is essentially a closed synchronization system (CCS), and the dependencies presented in the paper do not allow to evaluate the open-loop systems that belong to another class of synchronization systems.

In such scientific works as $[4,5]$, studies are described, aimed mainly at optimizing the parameters of the filter and the system as a whole for the class of closed synchronization systems (CCS). However, due to their inherent contradictions, the CCS does not allow in some cases to ensure the required quality of work. This is especially noticeable when it is necessary to improve the quality of the system on two or more conflicting indicators [6, 7].

There are great opportunities to improve the quality of synchronization systems in the class of combined synchronization systems (CSS). These systems can combine the principles of regulation of deflection and perturbation with the simultaneous provision of minimization of the phase error variance, which was defined as a promising method of constructing CSS and covered in [3, 6].

In [8], the peculiarities of the implementation of the carrier frequency recovery system with coherent demodulation of the signal with a continuous phase are shown. The scheme of construction of such system of phase autotuning of frequency is essentially the combined system of synchronization with feedback. The question of practical realization of the system of phase autotuning of frequency on a modern element base is investigated. But research on the possibilities of the scheme of construction of the synchronization system to increase astatism and reduce the variance of the assessment due in this article is missing.

The authors of $[9,10]$ proposed a method of implementing a certain type of signal sequence synchronization in CSS, which expands under conditions of significant excess of the noise level over the level of the information signal. For synchronization the service channel which works on one frequency with information is used. Channel distribution is performed during the formation of signals of quadrature channels: in-phase channel is used to generate a phase-locked signal with spread spectrum, quadrature channel is used to transmit a clock signal. The possibility of increasing astatism and ensuring the speed of the system in this work is not considered.

The article [11] presents a new direct sequence modulation scheme for distributed spectrum communication systems, defined as delay and addressing modulation (DADS). The scheme proposed by the authors is easy to implement and 
does not require alignment of the input signal code at its input, which makes it the most optimal for the transmission of short signals. The article does not disclose the type of scheme in relation to which the conclusions were substantiated, and there is no question of increasing the order of astatism in a particular scheme.

Certain studies on the possibilities of minimizing the variance of the phase error in the open-loop CSS and one of the options for constructing the CSS, which has such a combination along with the high speed of the system are presented in [12]. This paper develops and presents a mathematical model of CSS with an open connection of a certain type and evaluates its ability to minimize the phase error in the order of astatism not higher than the second. It is directly established that the open channel is made in the form of parallel (serial) connection of two links of the frequency discriminator with the proposed transfer function allows to increase the order of astatism to the third and higher order and does not affect the stability of the system. The paper shows that the effect on the variance of the phase error of the synchronization system, as substantiated in this paper, can be achieved by changing the parameters of the open link of the synchronization scheme of the system.

But in this paper there is no direct assessment of the possibilities of minimizing the root mean square error by means of an open (compensating) connection on the input signal by changing its parameters.

\section{Methods of research}

The block diagram of the linear model of the CSS synchronization system, which is considered in the work, is shown in Fig. 1. The structure of the specified model CSS includes an additional link with the transfer function $W_{4}(S)$ by means of which the open communication is carried out and the open control channel is formed [13].
Coherent reception requires accurate knowledge of the current phase of the carrier oscillation. When using a synchronization system as a phase filter, the input signal is, according to expression (1), the sum $d(t)+\Delta \psi(t)$, where $\Delta \psi(t)=\psi_{1}(t)-\psi_{2}(t), \psi_{2}(t)$ is the sum of various internal interferences. Processes $M(t), N(t)$ also represent in this case the general obstacle.

The phase error variance thus consists of four components [14]:

$$
\sigma_{\varphi}^{2}=\sigma_{d}^{2}+\sigma_{\Delta \varphi}^{2}+\sigma_{M}^{2}+\sigma_{N}^{2}
$$

where $\sigma_{d}^{2}$ - variance of the error on the dynamics of signal transmission; $\sigma_{\Delta \varphi}^{2}$ - variance of the phase shift of the carrier frequency; $\sigma_{M}^{2}-$ signal modulation error variance; $\sigma_{N}^{2}-$ variance of the error from the action of the internal noise of the system.

Each of which according to spectral theory is defined as follows:

$$
\begin{aligned}
& \sigma_{1}^{2}=\sigma_{d}^{2}+\sigma_{\Delta \varphi}^{2}=\frac{1}{2 \pi} \int_{-\infty}^{\infty}\left|W_{\varphi}(j \omega)\right|^{2} G_{S}(\omega) \mathrm{d} \omega, \\
& \sigma_{2}^{2}=\sigma_{M}^{2}+\sigma_{N}^{2}=\frac{1}{2 \pi} \int_{-\infty}^{\infty}\left|W_{\varphi}(j \omega)\right|^{2} G_{n}(\omega) \mathrm{d} \omega,
\end{aligned}
$$

where $W_{\varphi}(j \omega)$ - the transfer function of the synchronization system in the frequency domain; $G_{S}(\omega)$ - energy spectrum of signals at the input of the system.

To determine the components of phase error (2) let's use expressions (3) and (4) substituting in them the transfer functions for $\operatorname{CSS} W_{\varphi K}(S)=D_{\varphi K}(S) / F_{3}(S), W_{K}(S)=$ $=D_{K}(S) / F_{3}(S)$ and energy spectra $G_{S}(\omega)=G_{M}(\omega), G_{n}(\omega)=$ $=G_{N}(\omega)[12,15]$.

For ease of integration, let's present the integrals (3), (4) in the form of Perseval integrals [16]:

$$
I_{n}=\frac{1}{2 \pi} \int_{-\infty}^{\infty} \frac{M(j \omega)}{H(j \omega)-H(j \omega)} \mathrm{d} \omega
$$

where

$$
\begin{aligned}
& H(j \omega)=\sum_{i=0}^{n} \alpha_{i}(j \omega)^{n-i}, \\
& M(j \omega)=\sum_{i=0}^{n-1} \beta_{i}(j \omega)^{2(n-i-1)} .
\end{aligned}
$$

Fig. 1. Block diagram of a linear model of a combined synchronization system with an additional link

Then the values of the integrals $I_{n}$ are expressed by subtractions through coefficients $a_{i}, \beta_{i}$ as follows [16, 17]:

$$
I_{n}=\frac{\left[(-1)^{n+1} B_{n}\right]}{\left(2 \alpha_{0} A_{n}\right)},
$$

turn to the development of mathematical dependencies that allow to determine the phase error variance of the combined synchronization system under the influence of additive Gaussian noise.

In the general case, the phase modulation of the signal contains four components [14]:

$$
\varphi_{\text {input }}(t)=d(t)+M(t)+\Delta \psi(t)+N(t)
$$

where $d(t)$ - Doppler shift at the input; $M(t)$ - useful angular modulation; $\Delta \psi(t)$ - the total amount of obstacles. where

$$
A_{n}=\left|\begin{array}{cccc}
\alpha_{1} & \alpha_{0} & \ldots & 0 \\
\alpha_{0} & \alpha_{2} & \ldots & 0 \\
\ldots & \ldots & \ldots & \ldots \\
0 & 0 & \ldots & \alpha_{n}
\end{array}\right|,
$$


$A_{n}$ - Hurwitz senior determinant for the polynomial $H_{n}(j \omega)$; $B_{n}$ - determinant derived from the determinant $A_{n}$ by replacing the first column with coefficients $\beta_{i}$.

Expressions (5) and (6) will be useful in the future in the development of the algorithm and the analysis and synthesis of the synchronization system using computer simulation.

In what follows, let's consider a modulating signal $m(t)$ of two types: with a «maximally flat (butterworth) shape of the spectrum» and asymptotic Gaussian processes.

Their energy spectra can be described according to the following expressions [14]:

$$
\begin{aligned}
& G_{1}(\omega, n)=\frac{K_{1}(n)}{\left[1+\left(\frac{\omega}{\omega_{c}}\right)^{2 n}\right]}, \\
& G_{2}(\omega, n)=\frac{K_{2}(n)}{\left[1+\left(\frac{\omega}{\sqrt{n_{\omega_{c}}}}\right)^{2 n}\right]^{n}}, \quad n=1,2,3 \ldots
\end{aligned}
$$

where

$$
\begin{aligned}
& K_{1}(n)=\frac{\pi \sin c\left(\frac{\pi}{2 n}\right)}{\omega_{c}}, \\
& K_{2}(n)=\frac{(4 \pi)}{\left[\sqrt{n_{\omega_{c}}}\right]}, \quad \sin c=\sin x / x,
\end{aligned}
$$

$\omega_{c}$ - frequency corresponding to half the power.

The following spectra are characteristic of a wide class of signals used in communication [14]:

$$
G_{1}(\omega, 1)=G_{2}(\omega, 1)=G(\omega, 1),
$$

when $n=1$ they are the same.

Consider the possibility of reducing the root mean square error (RMS), which is associated with the minimum marginal variance of the phase error when tracking the carrier frequency in the CSS. Let's consider for a specific type of open communication link with the transfer function $W_{4}(S)$ and a fixed value of the parameter $\mathrm{n}$ in expressions (7) and (8) of the energy spectrum of the input signal. For this:

- develop analytical dependencies that allow to assess the degree of reduction of RMSE in CSS in comparison with closed synchronization system and the effectiveness of the introduction of open communication at different values of the signal/interference ratio;

- develop an algorithm for the synthesis of open communication for CSS;

- with the help of mathematical modeling on the basis of the developed algorithm let's study the change of UPC at different signal spectra and levels of additive interference at the system input.

Let's turn to the development of analytical dependences that allow to assess the degree of reduction of RMSE in CSS in comparison with closed synchronization system and the effectiveness of the introduction of open communication at different values of the signal-to-noise ratio.

Assume that as an open channel used a frequency discriminator with a transfer function of the form (9) at $n=1$, that is [18]:

$$
W_{4}(S)=\frac{\left(K_{4} S\right)}{\left(T_{4} S+1\right)}
$$

The parameter $n$ in expressions (7) and (8) is taken equal to one. With:

$$
G_{1}(w)=G_{2}(w)=\frac{\left(\frac{2}{w_{c}}\right)}{\left[1+\left(\frac{w}{w_{c}}\right)^{2}\right]} .
$$

Let's consider the case when the main interfering factor of the system is additive Gaussian noise. The spectrum of phase modulation is due only to the signal $m(t)$ and is equal to $[14,15]$ :

$$
G_{M}(\omega)=K_{m}^{2} G_{i}(\omega)
$$

Since this paper considers high-precision synchronization systems, let's assume that the magnitude of the phase error (or its variance) satisfies the conditions of smallness [15, 17], which allows to consider it as a linear model. If the input noise is defined as «white» and with a one-way spectral density $N_{0} / 2$, then as shown in $[15,16]$, the equivalent phase noise $N_{\varphi}$ is also approximately «white».

The energy spectrum of the reduced phase noise, listed at the input of the system, will be:

$$
G_{N}(\omega)=\frac{N_{0}}{\left(2 A_{0}^{2}\right)} .
$$

Let's define the expression of the variance of the phase error. For this:

- substitute in expressions (3) and (4) the transfer functions of CSS [12, 15];

- take into account the energy spectra of the input phase of the vortex signal. (10) and (5) and the energy spectra of the equivalent phase noise listed at the input (11)

- use relations (5) and (6).

The final expression of the phase error variance, taking into account the requirements presented in [13], will look like:

$$
\sigma_{\varphi K}^{2}=\sigma_{1} K_{4}^{2}+\sigma_{2} K_{4}+\sigma_{3} .
$$

Let's give expression (12) as follows:

$$
\sigma_{\varphi K}^{2}=\sigma_{1}\left[\left(K_{4}+\frac{\delta_{2}}{2 \delta_{1}}\right)^{2}+\frac{4 \delta_{1} \delta_{3}-\delta_{2}^{2}}{4 \delta_{1}}\right] .
$$

From this relation it follows that the function $\sigma_{\varphi K}^{2}$ has a maximum in the real domain at the optimal value of $K_{4}$, which is determined by the following expression:

$$
K_{4}=\frac{\delta_{2}}{\left(2 \delta_{1}\right)}, \quad \delta_{2}<0 .
$$

In this case, the minimum value of the phase error variance is equal to $[13,15]$ :

$$
\sigma_{\varphi K_{\min }}^{2}=\frac{\delta_{3}-\delta_{2}^{2}}{\left(4 \delta_{1}\right)}=\sigma_{\varphi 3}^{2}-\Delta \sigma_{\varphi}^{2} .
$$


For a closed system, the minimum value of the error variance can be obtained from expression (12) at $K_{4}=0$ :

$$
\sigma_{\varphi 3 \min }^{2}=\delta_{3} .
$$

Since $\delta_{1}>0$ and $\delta_{1}^{2}>0$, the value of the error variance in CSS (15) is less than in closed synchronization system (12) by the value $\Delta \delta_{\varphi}^{2}=\delta_{2}^{2} / 4 \delta_{1}$ of any choice of parameters of the latter.

In order for the minimum of function (13) to be in the region of positive values of parameter $K_{4}$, it is necessary that the equality:

\section{$\delta_{2}>0$.}

Let's consider what determines the value of the parameter $\delta_{2}$ and, through it, the scope of CSS.

In general $[15,16]$

$$
\begin{aligned}
& \delta_{2}=C_{2}\left(\omega_{c}\right)\left[\frac{\left(A_{0} K T_{1}\right)}{\left(2 a_{0} q\right)}-K_{m}^{2} F_{1}\left(\omega_{c}\right)\right] \\
& C_{2}\left(\omega_{c}\right)=\frac{\left(2 A_{0} K_{3}\right)}{\left(a_{1} \omega_{c}\right)}>0 ; \quad \frac{\left(A_{0} K T_{1}\right)}{\left(2 a_{0} q\right)}>0 ; \\
& F_{1}\left(\omega_{c}\right)=a_{0}+a_{1} \omega_{c}^{-1}+a_{2} \omega_{c}^{-2}>0 ; \quad F_{1}\left(\frac{P_{C}}{P_{N_{s}}}\right)=\frac{\left(\omega_{c} N_{0}\right)}{\left(2 A_{0}^{2}\right)}=q^{-1} .
\end{aligned}
$$

The analysis of the given relations shows that at $T_{1}=0$, (i. e. at a closed circuit the aperiodic link is used as the filter), condition (17) is fulfilled at any values of parameters of system and signals therefore CSS gives advantage in comparison with closed synchronization system.

At $T \neq 0$ it is necessary that the inequality [16] holds:

$$
\frac{\left(A_{0} K T_{1}\right)}{\left(2 a_{0} q\right)}<K_{m}^{2} F_{1}\left(\omega_{c}\right)
$$

If to take into account that $T_{1}\left(2 T_{2}\right)=m / 2$, where $m \leq 1$ is a filter parameter [14, 15], then inequality (18) in the expanded form will be:

$$
\left(A_{0} K T_{1}\right)<\frac{\left(2 q T_{2} \omega_{c}^{2} K_{m}^{2}\right)}{\left(a_{0} \omega_{c}^{2}+a_{1} \omega_{c}+a_{2}\right)} .
$$

Additional reduction of the phase error variance in CSS is possible, as shown in [12] due to the appropriate choice of the denominator parameter of open communication.

6.2. Estimation of influence of parameters of open link on minimization of phase error dispersion of combined synchronization system from signal level of additive Gaussian noise. The graphs of dependence $\eta=\delta_{\phi K}^{2} / \delta_{\phi 3}^{2}=f\left(K_{4}, T_{4}\right)$, obtained by the method of mathematical modeling with the specification of their dependencies according to the works [6, 19] are given in Fig. 2.

As follows from these dependencies, increasing the parameter $T_{4}$ reduces the value $\delta_{\varphi K}^{2}$ and causes a change in the optimal value of the parameter $K_{4}$.

But an increase in the parameter $T_{4}$ is equivalent to a decrease in the introduced open-bonded root. Therefore, the change $T_{4}$ is limited to the smallest value of the root of the characteristic equation of a closed system. $\eta=\delta_{\varphi K}^{2} / \delta_{\varphi 3}^{2}$

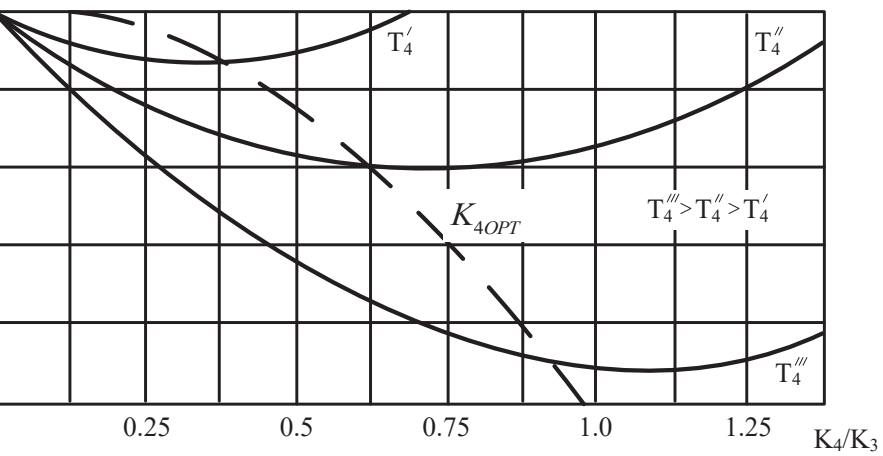

Fig. 2. The dependence of the variance of the phase error of the C5S on the parameters of the open channel

As the parameter $n$ in formulas (7), (8) increases, the expressions for the phase error variance become more complicated and their direct analysis becomes difficult.

The final expression for the variance of the phase error in this case can be written as follows, taking into account the requirements of $[13,15,16]$ :

$$
\delta_{\varphi K}^{2}=N_{1} F_{1}\left[\beta_{22}\left(K_{4}\right)\right]+N_{2} F_{2}\left[\beta_{10}\left(K_{4}\right)\right]
$$

where function from the phase modulation index by a useful process:

$$
\begin{aligned}
& N_{1}=\frac{\left(b K_{t}^{2}\right)}{\left[2\left(a_{20} a_{23}^{2}+a_{21}^{2} a_{24}-a_{21} a_{22} a_{23}\right)\right]}=f_{1}\left(K_{m}\right), \\
& N_{2}=\frac{N_{0}}{4 A_{0}^{2} a_{0} a_{1} a_{2}}=f_{2}\left(N_{0}\right), \\
& F_{1}\left[\beta_{22}\left(K_{4}\right)\right]=f_{3}\left(K_{4}\right), F_{2}\left[\beta_{10}\left(K_{4}\right)\right]=f_{4}\left(K_{4}\right) .
\end{aligned}
$$

The optimal value of the parameter $K_{4}$ that minimizes the function (20) will be [16]:

$$
K_{4 O P T}=\frac{\left(\alpha_{21} N_{1} K_{3}+a_{2} N_{2} K_{1}^{2} K_{3}\right)}{\left(\alpha_{21} N_{1} K_{3}^{20}+a_{2} N_{2} K_{1}^{2}\right)} .
$$

Substitution (21) in (20) gives the minimum value of the variance of the phase error in the CSS:

$$
\sigma_{\varphi K_{\min }}^{2}=\sigma_{\varphi 3}^{2}-\Delta \sigma_{\varphi}^{2},
$$

where

$$
\begin{aligned}
& \sigma_{\varphi}^{2}=N_{2}\left(a_{0} \beta_{11}-a_{0} \beta_{11}\right)-N_{12}\left(\alpha_{21}-\alpha_{23} \beta_{21}\right), \\
& \Delta \sigma_{\varphi}^{2}=\frac{\left(\alpha_{21} N_{1} K_{3}-a_{2} N_{2} K_{1}^{2} K_{3}\right)}{2}>0 .
\end{aligned}
$$

That is, in this case, the variance of the phase error in CSS is also less than in closed synchronization system by value $\Delta \sigma_{\varphi}^{2}$.

In this case, the effect of reducing the variance of the phase error due to the introduction of communication at a given influence depends on the noise level in the channel (function $N_{2}=f_{2}\left(N_{0}\right)$ ) and the phase modulation index of the input signal process (8) (function $N_{1}$ ) from the signal power.

The results of CSS analysis using mathematical modeling are reflected in the dependencies Fig. 3, 4. 


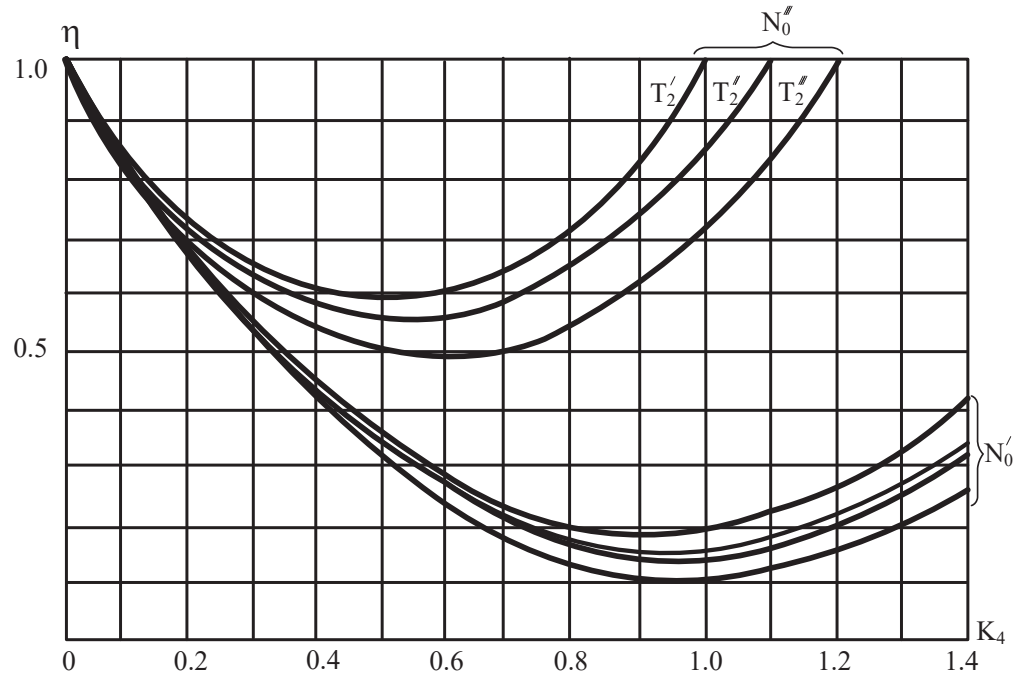

Fig. 3. Dependencies $\eta=f_{2}\left(K_{4}, T_{2}, N_{0}=\right.$ const $)$

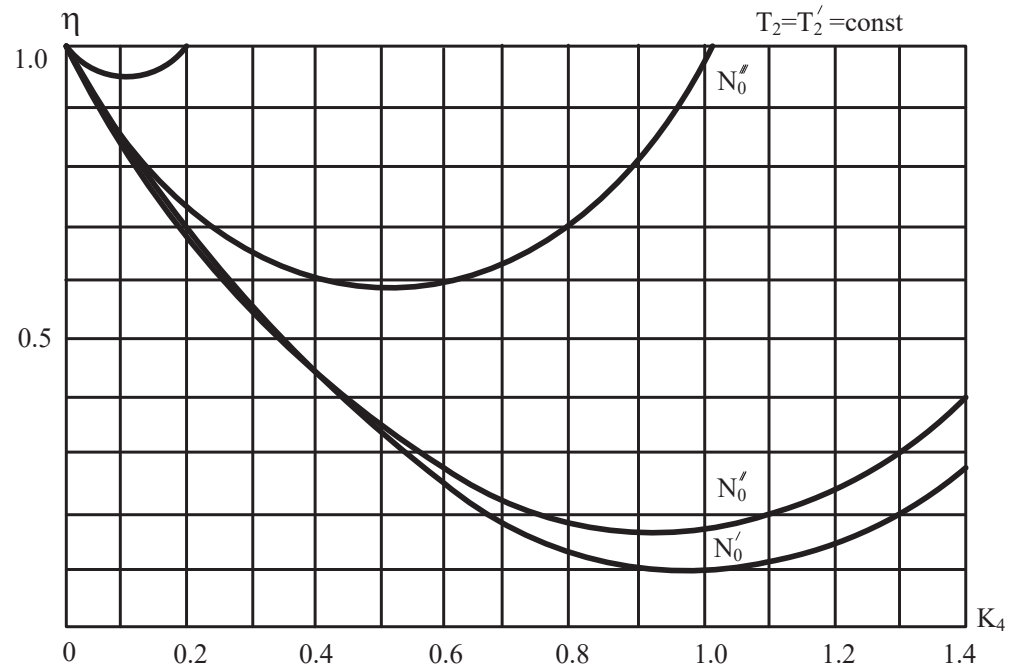

Fig. 4. Dependencies $\eta=f_{1}\left(K_{4}, N_{0}, T_{2}=T_{4}=\right.$ const $)$

Analysis of Fig. 3 and Fig. 4 shows that at a certain obstacle level, an open link becomes ineffective.

\section{SWOT-analysis of research results}

Strengths. In comparison with analogues, the positive effect of the object of study allows to evaluate the possibilities of the scheme of the combined synchronization system directly considered in the article to minimize the phase error variance in certain conditions of additive Gaussian and other types of noise. And the proposed mathematical dependencies have the potential to improve the algorithm of this estimate for the implementation of such in relation to other schemes of CSS with open connection.

Weaknesses. The weaknesses of the work include a detailed consideration and assessment of the possibilities of only one of the simple implementations of the combined synchronization system with open (compensating) connection. Other schemes of simple realization of such communication, and also synthesis of various types of simple communication in one scheme of realization in work are not considered.

Their analysis shows that at a certain level of interference, open communication becomes ineffective.
Opportunities. A further promising study in the direction of solving a significant scientific problem in the article is to assess the possibilities for minimizing the variance of the phase error under the influence of noise in the synthesis of more complex open-circuit schemes, for which it is necessary to create appropriate mathematical models and estimation algorithms.

Threats. The scientific results of the research can be used in the development of combined phase synchronization systems with a simple open connection, which can operate in conditions of external noise not higher than a certain level of their signal. The development of more noise-tolerant and noise-protected synchronization systems requires further research to create more complex open-circuit schemes and evaluate their ability to minimize phase error variance under external noise with a given signal level.

\section{Conclusions}

1. Mathematical dependencies, allowing to determine the variance of the phase error under the influence of additive Gaussian noise are developed and refined. And also the corresponding algorithm for its determination is submitted. By selecting the parameters of the constituent elements of the circuit for constructing a combined synchronization system with an open (compensating) coupling, an assessment is made of the possibilities of minimizing the phase error variance under the influence of additive Gaussian noise

2. Owing to the assessment of the influence of the parameters of the open-loop link on the variance minimization of the phase error of the combined synchronization system from the signal level of the additive Gaussian noise, it is shown that:

- the value of the error variance for the combined synchronization system is much less than for the closedloop synchronization system for any choice of the parameters of the latter;

- the effect of reducing the variance of the phase error due to the introduction of a broken connection according to a given influence depends on the level of the noise signal and on the modulation index of the phase of the input signal

- when used as a simple broken connection of a frequency discriminator included in the scheme of a combined synchronization system in parallel, the minimum variance of the phase error with an increase in the noise level to a certain critical point has limitations, and this broken connection loses its effectiveness.

\section{References}

1. Boiko, Yu. M., Nochka, R. Yu. (2015). Otsiniuvannia yakisnykh pokaznykiv prystroiv synkhronizatsii syhnaliv zasobiv telekomunikatsii. Visnyk Khmelnytskoho natsionalnoho universytetu, 1, 144-155.

2. Mucchi, L., Marabissi, D., Ranaldi, M., Del Re, E., Fantacci, R. (2004). Impact of synchronization errors and multiple access 
interference to the performance of UWB impulse radio systems. Eighth IEEE International Symposium on Spread Spectrum Techniques and Applications - Programme and Book of $\mathrm{Ab}$ stracts (IEEE Cat. No.04TH8738). Sydney, 477-483. doi: http:// doi.org/10.1109/isssta.2004.1371746

3. Shakhtarin, B. I. (2016). Analiz sistem sinkhronizatsii pri nalichii pomekh. Moscow: Goriachaia liniia - Telekom, 360.

4. Glukhov, A. V. (2013). Optimizatsiia parametrov tsifrovykh filtrov vysokoskorostnogo moduliatora dlia PLC-modemov. Vestnik Tambovskogo gosudarstvennogo tekhnicheskogo universiteta, 19 (4), 751-756

5. Lyons, R. G. (2010). Understanding Digital Signal Processing. Boston: Prentice Hall, 992.

6. Scheers, B., Le Nir, V. A. (2010). Modified Direct-Sequence Spread Spectrum Modulation Scheme for Burst Transmissions. Military Communications and Information Systems Conference (MCC'2010). Wroclaw, 366-373.

7. Turovskyi, O. L. (2019). Minimizatsiia dyspersii fazovoi pomylky v systemakh fazovoi synkhronizatsii zamknutoho typu $\mathrm{v}$ rezhymi stezhennia za nesuchoiu chastotoiu. Visnyk inzhenernoi akademii, 4, 22-27.

8. Kucher, D. B., Makohon, V. P. (2013). Vidnovlennia nesuchoi pry koherentnii demoduliatsii syhnalu z bezperervnoiu fazoiu zasobiv zviazku. Nauka i tekhnika Povitrianykh Syl Zbroinykh Syl Ukrainy, 2 (11), 148-149.

9. Kay, S. (1989). A fast and accurate single frequency estimator. IEEE Transactions on Acoustics, Speech, and Signal Processing, 37 (12), 1987-1990. doi: http://doi.org/10.1109/29.45547

10. Tikhomirov, A. V., Omelianchuk, E. V., Semenova, A. Iu. (2019) Sinkhronizatsiia $\mathrm{v}$ sistemakh s priamym rasshireniem spektra. Inzhenernii vestnik Dona, 9, 31-35.

11. Le Nir, V., van Waterschoot, T., Moonen, M., Duplicy, J. (2009). Blind CP-OFDM and ZP-OFDM Parameter Estimation in
Frequency Selective Channels. EURASIP Journal on Wireless Communications and Networking, 2009 (1). doi: http://doi.org/ $10.1155 / 2009 / 315765$

12. Turovsky, O., Khlaponin, Y., Muhi-Aldin, H. M. et. al. (2020) Combined system of phase synchronization with increased order of astatism in frequency monitoring mode. CEUR Workshop Proceedings, 2616 (1), 53-62.

13. Horowitz, P., Hill, W. (2015). The Art of Electronics. Cambridge: Cambridge University Press, 1220.

14. Sklar, B. (2017). Digital Communications: Fundamentals and Applications Second Edition. Prentice Hall, 1104.

15. Bondarenko, V. N. (2015). Pomekhoustoichivost priema spektralno - effektivnykh shumopodobnykh signalov. Krasnoiarsk: Sibirskii Federalnii universitet, 160.

16. Leman, E. (1991). Teoriia tochechnogo otsenivaniia. Moscow: Nauka, 448

17. Zalmanzon, L. A. (1989). Preobrazovaniia Fure, Uolsha, Khaara $i$ ikh primenenie v upravlenii, sviazi $i$ drugikh oblastiakh. Moscow: Nauka, 496

18. Misrikhanov, M. Sh. (2003). Invariantnoe upravlenie mnogomernymi sistemami. Moscow: Energotomizdat, 236.

19. Marey, M., Steendam, H. (2007). Analysis of the Narrowband Interference Effect on OFDM Timing Synchronization. IEEE Transactions on Signal Processing, 55 (9), 4558-4566. doi: http:// doi.org/10.1109/tsp.2007.896020

Turovsky Olexandr, PhD, Associate Professor, Head of Department of Organization of Training and Certification of Postgraduate and Doctoral Students, State University of Telecommunications, Kyiv, Ukraine, e-mail: s19641011@ukr.net, ORCID: http://orcid.org/ 0000-0002-4961-0876 